Published in final edited form as:

Science. 2019 March 29; 363(6434): 1463-1467. doi:10.1126/science.aaw1219.

\title{
Slide-seq: A Scalable Technology for Measuring Genome-Wide Expression at High Spatial Resolution
}

\author{
Samuel G. Rodriques ${ }^{1,2,3, \dagger}$, Robert R. Stickels ${ }^{3,4,5, \dagger}$, Aleksandrina Goeva $^{3}$, Carly A. Martin ${ }^{3}$, \\ Evan Murray ${ }^{3}$, Charles R. Vanderburg ${ }^{3}$, Joshua Welch ${ }^{3}$, Linlin M. Chen ${ }^{3}$, Fei Chen ${ }^{\star}, 3,{ }^{\dagger}$, \\ Evan Z. Macosko*,3,6,†† \\ ${ }^{1}$ Department of Physics, Massachusetts Institute of Technology, Cambridge, MA, 02139. \\ ${ }^{2}$ MIT Media Lab, Massachusetts Institute of Technology, Cambridge, MA, 02139. \\ ${ }^{3}$ Broad Institute of Harvard and MIT, Cambridge, MA, 02142. \\ ${ }^{4}$ Graduate School of Arts and Sciences, Harvard University, Cambridge, MA, 02138. \\ ${ }^{5}$ Division of Medical Science, Harvard Medical School, Boston, MA, 02115. \\ ${ }^{6}$ Department of Psychiatry, Massachusetts General Hospital, Boston, MA, 02114.
}

\begin{abstract}
Spatial positions of cells in tissues strongly influence function, yet a high-throughput, genomewide readout of gene expression with cellular resolution is lacking. We developed Slide-seq, a method for transferring RNA from tissue sections onto a surface covered in DNA-barcoded beads with known positions, allowing the locations of the RNA to be inferred by sequencing. Using Slide-seq, we localized cell types identified by scRNA-seq datasets within the cerebellum and hippocampus, characterized spatial gene expression patterns in the Purkinje layer of mouse cerebellum, and defined the temporal evolution of cell-type-specific responses in a mouse model of traumatic brain injury. These studies highlight how Slide-seq provides a scalable method for obtaining spatially resolved gene expression data at resolutions comparable to the sizes of individual cells.
\end{abstract}

\section{One Sentence Summary:}

\footnotetext{
"Correspondence to: chenf@broadinstitute.org, emacosko@broadinstitute.org.

$\dagger$ These authors contributed equally to this work.

${ }^{\dagger}$ These authors contributed equally to this work.

Author Contributions: F.C. and E.Z.M. conceived of the idea and supervised the work. S.G.R. and R.R.S. developed the puck fabrication methods. S.G.R. developed the puck sequencing and base-calling pipeline. S.G.R. and E.M. made the sequenced pucks. R.R.S. developed the tissue processing and library preparation pipeline. S.G.R and R.R.S. performed experiments generating data for the manuscript, with assistance from C.A.M., L.M.C., and C.V. A.G. developed the NMFreg method and performed the associated analyses with R.R.S and S.G.R. J.W. performed some initial analyses to help motivate using NMF for spatial mapping of cell types. S.G.R. developed the analysis pipelines for identifying spatially non-random and significantly correlated genes. S.G.R., R.R.S., F.C., and E.Z.M. wrote the manuscript.
}

Data Availability Statement: Raw image data of pucks, raw sequencing data, processed Slide-seq data, and NMFreg dependencies are available at the Broad institute's single-cell repository (https://portals.broadinstitute.org/single_cell/study/slide-seq-study). Code used to analyze the data is available with a detailed README in a Zenodo repository (DOI: 10.5281/zenodo.2571615). An up-to-date version of the analysis code, along with an up-to-date README, is available as a Github repository (https://github.com/broadchenf/ Slideseq).

Competing Interests: S.G.R., R.R.S., C.A.M., F.C. and E.Z.M. are listed as inventors on a patent application relating to the work. 
Slide-seq measures genome-wide expression in complex tissues at 10-micron resolution.

The functions of complex tissues are fundamentally tied to the organization of their resident cell types. Multiplexed in situ hybridization and sequencing-based approaches can measure gene expression with subcellular spatial resolution $(1,2)$, but require specialized knowledge and equipment, as well as the upfront selection of gene sets for measurement. By contrast, technologies for spatially encoded RNA-sequencing with barcoded oligonucleotide capture arrays are limited to resolutions in the hundreds of microns (3), which is insufficient to detect important tissue features.

To develop Slide-seq for high-resolution genome-wide expression analysis, we first packed uniquely DNA-barcoded $10 \mu \mathrm{m}$ microparticles ('beads') — similar to those used in the Dropseq approach to scRNA-seq (4)—onto a rubber-coated glass coverslip forming a monolayer we termed a "puck" (Fig. S1). We found that each bead barcode sequence could be uniquely determined via SOLiD sequencing-by-ligation chemistry (Figs. 1A, S1) (5-7). We next developed a protocol wherein $10 \mu \mathrm{m}$ fresh-frozen tissue sections were transferred onto the dried bead surface via cryosectioning (6). mRNA released from the tissue was captured onto the beads for preparation of 3'-end, barcoded RNA-seq libraries (4) (Fig. 1B). Clustering of individual bead profiles from a coronal section of mouse hippocampus (6) yielded assignments reflecting known positions of cell types in the tissue (Fig. 1C). Very fine spatial features were resolved, including the single-cell ependymal cell layer between the central ventricle and the habenula in the mouse brain (Fig. 1C, inset). Moreover, Slide-seq could be applied to a range of tissues, including the cerebellum and olfactory bulb, where layered tissue architectures were immediately detectable (Fig. 1D, S2), as well as liver and kidney, where the identified clusters revealed hepatocyte zonation patterns (8) and the cellular constituents of the nephron, respectively. Slide-seq on postmortem human cerebellum was also successful in capturing the same architectural features observed in the cognate mouse tissue (Fig. S3). Expression measurements by Slide-seq agreed with those from bulk mRNAseq and scRNA-seq, and average mRNA transcript capture per cell was consistent across tissues and experiments (Fig. S4). Finally, we found no detectable difference in the dimensions of brain structures observed in Slide-seq and in FISH (Fig. S5), implying that mRNA is transferred from the tissue to the beads with minimal lateral diffusion.

To map scRNA-seq cell types onto Slide-seq data, we developed a computational approach called Non-negative Matrix Factorization Regression (NMFreg) that reconstructs expression of each Slide-seq bead as a weighted combination of cell-type signatures defined by scRNAseq (Fig. 2A). Application of NMFreg to a coronal mouse cerebellar puck recapitulated the spatial distributions of classical neuronal and non-neuronal cell types (9), such as granule cells, Golgi interneurons, unipolar brush cells, Purkinje cells, and oligodendrocytes (Fig. 2B, S6A). The mapping by NMFreg was found to be reliable across a range of factor numbers and random restarts (Fig. S6B,C). We found that $65.8 \%+/-1.4 \%$ of beads could be identified with a single cell type (6), whereas $32.6 \%+/-1.2 \%$ showed mRNA from two cell types (mean \pm std, $\mathrm{N}=7$ cerebellar pucks) (Figs. 2C, S7). The high spatial resolution of Slide-seq was key to mapping cell types: when data were aggregated into larger feature sizes, cell types in heterogeneous regions of tissue could not be resolved (Fig. S8). Slide-seq 
collects a 2D spatial sample of 3D tissue volumes, thus caution should be taken when making absolute counting measurements throughout the $3 \mathrm{D}$ volume in the absence of proper stereological controls and sampling methods (10).

We first sequenced pucks capturing 66 sagittal tissue sections from a single dorsal mouse hippocampus (20 billion paired-end reads over 1.5 million barcoded beads), covering a volume of 39 cubic millimeters, with roughly $10 \mu \mathrm{m}$ resolution in the dorsal-ventral and anterior-posterior axes, and $\sim 20 \mu \mathrm{m}$ resolution (alternate $10 \mu \mathrm{m}$ sections) in medial-lateral axis (Fig. S9A-D). Using NMFreg, 770,000 beads in the volume could be associated with a single scRNA-seq-defined cell type. We computationally co-registered pucks along the medial-lateral axis, allowing for visualization of the cell types and gene expression in the hippocampus in three dimensions (Fig. 2D,S9E,F, Supplementary Video 1). We plotted metagenes comprised of previously defined markers (9) for the dentate gyrus, CA2, CA3, a subiculum subpopulation, an anteriorly localized CA1 subset (exemplified by the marker Tenm3) and cells undergoing mitosis and neurogenesis. The metagenes were highly expressed and specific for the expected regions (Fig. 2E), confirming the ability of Slide-seq to localize both common cell-types as well as finer cellular subpopulations. The entire experimental processing of these 66 pucks (excluding puck generation) required $\sim 40$ personhours (6), and only standard experimental apparatus.

We then developed a nonparametric, kernel-free algorithm to identify genes with spatially non-random distribution across the puck (Fig. S10) (6). Application of this algorithm to coronally sliced cerebellum identified Ogfrl1, Prkcd and Atp2b1 as highly localized to a region just inferior to the cerebellum (Fig. S11A). We found $O g f r 11$ in particular to be a specific and novel marker for PV interneurons in the molecular and fusiform layers of the dorsal cochlear nucleus (Fig. S11B), likely the cartwheel cells of the dorsal cochlear nucleus $(11,12)$. Our algorithm also identified Rasgrf1 as expressed only in granule cells anterior to the primary fissure (Fig. S11C, cyan, S11D, left) (13), and further analysis revealed four previously uncharacterized genes expressed only posterior to the primary fissure (6) (Table S2, Fig. S11C, yellow, Fig. S11D, right).

The cerebellum is marked by parasagittal bands of gene expression in the Purkinje layer that correlate with heterogeneity in Purkinje cell physiology and projection targets (14-17).

Several genes, including Aldoc (also known as the antigen of the Zebrin II antibody) show similar or complementary parasagittal expression $(16,18,19)$ but the extent of this form of expression variation is unknown, and these patterns have not previously been identified in single-cell sequencing studies. Using the spatial information afforded by Slide-seq, we identified 669 spatially non-random genes in the Purkinje layer (Table S2), of which 126 appeared either correlated or anticorrelated with the Zebrin pattern, using Aldoc and Plcb4 as markers of Zebrin II(+) or Zebrin II(-) bands, respectively (Fig. 3A). Among the anticorrelated genes were four ATPases and four potassium channels, including some which may explain differences in electrophysiology between Zebrin II(+) and Zebrin II(-) Purkinje neurons (Table S2). Moreover, we identified several other patterns of spatial gene expression, besides the Zebrin pattern. While most genes identified displayed a pattern consistent with Zebrin II staining (Figs. 3B,C), several were exclusively expressed in or excluded from the vestibulocerebellar region (lobules IX and X) (20, 21) (Fig. 3D, Table 
S2), confirming that lobules IX and $X$ have a distinct program of gene expression. Still other genes showed either exclusive expression in (e.g. B3galt5 $(6,22))$ or exclusion from (e.g. Gnail) lobules IX/X and VI/VII (Fig. S11E,F), suggesting that these regions might share a pattern of gene expression, despite the disparate cognitive roles associated with them (23). Finally, although only Purkinje cells have previously been associated with the Aldoc pattern, we found that Mybpc1, a Bergmann cell marker previously only studied in the context of muscle, appears in both Slide-seq (Fig. S11G) and in situ data (Fig. S11H) to have a Zebrin pattern of expression. We thus conclude that the banded gene expression patterns divide many cerebellar cell types, including Purkinje cells, Bergmann glia, and granule cells, into spatially defined subpopulations, which was not indicated in previous single-cell sequencing studies $(9,24)$.

Finally, we applied Slide-seq to quantify the brain's response to traumatic brain injury over time. Cortical injuries were visualized in Slide-seq data by the presence of hemoglobin transcripts 2 hours after the injury (Fig. 4A), or by transcripts of Vim, Gfap, and Ctsd at 3 days and 2 weeks after the injury (Fig. 4B,C). Vim, Gfap, and Ctsd were chosen because they are known markers of the astrocytic (Vim and Gfap) or microglial (Vim and Ctsd) responses that were found to be highly upregulated at the injury in the Slide-seq data (Fig. S13). We applied an algorithm to identify all genes that correlate spatially with those transcripts. At the 2-hour timepoint, only Fos and rRNA (25) were found to correlate spatially with the injury (Figs. 4A, S14). By contrast, at the 3-day timepoint, we found microglia/macrophages-assigned beads localized to the injury, bordered by a distinct layer of cells (thickness: $92.4 \mu \mathrm{m} \pm 11.3 \mu \mathrm{m}$, mean \pm sterr, $\mathrm{N}=3$ ) expressing mitosis-associated factors, followed by a layer of astrocyte-assigned beads (Fig. 4D). Finally, at the 2-week timepoint, we observed microglia/macrophage-assigned beads filling the injury, surrounded by an astrocytic scar (thickness: $36.6 \mu \mathrm{m} \pm 13.4 \mu \mathrm{m}$, mean \pm sterr, $\mathrm{N}=6$ ), with evidence of microglia (but not macrophages) penetrating $39 \mu \mathrm{m} \pm 17.8 \mu \mathrm{m}$ (mean \pm sterr, $\mathrm{N}=6$ ) through the astrocytic scar and into neuron-rich regions (Fig. 4E,F). Macrophages were visualized using $L y z 2$, a specific marker for macrophages and granulocytes, however, we interpret this as a marker of macrophages, because other granulocyte-specific markers were not found to colocalize with Gfap, Ctsd, and Vim.

In order to investigate other changes in gene expression between the 3-day and 2-week timepoints, we identified genes that correlated spatially with Vim, Gfap, and Ctsd at the 3day timepoint or the 2-week timepoint (6). Applying gene ontology analysis to these gene sets revealed enrichment of annotations relating to chromatid segregation, mitosis, and cell division at the 3-day timepoint (Fig. 4G), and relating to the immune response (Fig. 4H), gliogenesis (Fig. 4I) and oligodendrocyte development (Fig. 4J) at the 2-week timepoint. This suggests that cell proliferation occurs in the first few days following injury, and transitions to differentiation on the order of weeks. For example, although the degree to which oligodendrocyte progenitor cells (OPCs) differentiate into oligodendrocytes following a focal gray matter injury is controversial (26), we confirmed that both Sox 4 and Sox 10 localize to the region surrounding the injury at the 2 week timepoint, indicating the presence of immature oligodendrocytes (Fig. S15). We also discovered evidence that several immediate early genes, including highly neuron-specific genes such as Npas4 (Table S2), are upregulated in a region of width $0.72 \mathrm{~mm} \pm 0.19 \mathrm{~mm}$ (mean \pm sterr, $\mathrm{N}=4$ measurements) 
around the injury at both the 3-day and the 2-week timepoints (27-29) (Fig. 4K, Table S2), suggesting persistent effects of the injury on neural activity in a large area around the injury.

Here we demonstrate that Slide-seq enables the spatial analysis of gene expression in frozen tissue with high spatial resolution and scalability to large tissue volumes. Slide-seq is easily integrated with large-scale scRNA-seq datasets and enables discovery of spatially defined gene expression patterns in normal and diseased tissues. The primary cost of Slide-seq is the cost of short read sequencing, which is $\sim \$ 200-\$ 500$ for the pucks presented here. As the cost of sequencing drops further, we expect to be able to scale Slide-seq to entire organs or even entire organisms. We anticipate that Slide-seq will play important roles in positioning molecularly defined cell types in complex tissues, and defining new molecular pathways involved in neuropathological states.

\section{Supplementary Material}

Refer to Web version on PubMed Central for supplementary material.

\section{Acknowledgments:}

We would like to acknowledge E. Boyden for his support. We would like to thank D. Goodwin and S. Alon for useful discussions relating to the identification of rRNA in single-cell sequencing data. We would like to thank J. Marshall and A. Greka for helpful discussions regarding kidney tissues. We would like to thank Alec Wysoker, Jim Nemesh, and Steve McCarroll for the use of their Drop-seq analysis pipeline. We acknowledge J. Bloom for help with development of methods, and V. Kozareva and T. Kamath for assistance with algorithm implementation.

Funding: This work was supported by an NIH New Innovator Award (DP2 AG058488-01), an NIH Early Independence Award (DP5, 1DP5OD024583), the Schmidt Fellows Program at the Broad Institute and the Stanley Center for Psychiatric Research. S.G.R. acknowledges funding through the Hertz Graduate Fellowship and the National Science Foundation Graduate Research Fellowship Program (award \#1122374).

\section{References and Notes}

1. Shah S, Lubeck E, Zhou W, Cai L, seqFISH Accurately Detects Transcripts in Single Cells and Reveals Robust Spatial Organization in the Hippocampus. Neuron. 94, 752-758.e1 (2017). [PubMed: 28521130]

2. Chen KH, Boettiger AN, Moffitt JR, Wang S, Zhuang X, Spatially resolved, highly multiplexed RNA profiling in single cells. Science. 348 (2015).

3. Stahl PL et al., Visualization and analysis of gene expression in tissue sections by spatial transcriptomics. Science. 353, 78-82 (2016). [PubMed: 27365449]

4. Macosko EZ et al., Highly parallel genome-wide expression profiling of individual cells using nanoliter droplets. Cell. 161, 1202-1214 (2015). [PubMed: 26000488]

5. Lee JH et al., Fluorescent in situ sequencing (FISSEQ) of RNA for gene expression profiling in intact cells and tissues. Nat. Protoc. 10, 442-58 (2015). [PubMed: 25675209]

6. Materials and methods are available as supplementary materials online.

7. Gunderson KL et al., Decoding Randomly Ordered DNA Arrays, 870-877 (2004).

8. Halpern KB et al., Single-cell spatial reconstruction reveals global division of labour in the mammalian liver. Nature. 542, 352-356 (2017). [PubMed: 28166538]

9. Saunders A et al., Molecular Diversity and Specializations among the Cells of the Adult Mouse Brain. Cell. 174, 1015-1030.e16 (2018). [PubMed: 30096299]

10. West MJ, New stereological methods for counting neurons. Neurobiol. Aging. 14, 275-285 (1993). [PubMed: 8367009]

11. Trussell LO, Oertel D, (Springer, Cham, 2018; http://link.springer.com/ 10.1007/978-3-319-71798-2_4), pp. 73-99. 
12. Roberts MT, Trussell LO, Molecular Layer Inhibitory Interneurons Provide Feedforward and Lateral Inhibition in the Dorsal Cochlear Nucleus. J. Neurophysiol. 104, 2462-2473 (2010). [PubMed: 20719922]

13. Lein ES et al., Genome-wide atlas of gene expression in the adult mouse brain. Nature. 445, $168-$ 176 (2007). [PubMed: 17151600]

14. Gravel C, Hawkes R, Parasagittal organization of the rat cerebellar cortex: Direct comparison of purkinje cell compartments and the organization of the spinocerebellar projection. J. Comp. Neurol. 291, 79-102 (1990). [PubMed: 1688891]

15. Xiao J et al., Systematic regional variations in Purkinje cell spiking patterns. PLoS One. 9, e105633 (2014).

16. Barmack NH, Qian Z, Yoshimura J, Regional and cellular distribution of protein kinase C in rat cerebellar Purkinje cells. J. Comp. Neurol. 427, 235-54 (2000). [PubMed: 11054691]

17. Cerminara NL, Lang EJ, V Sillitoe R, Apps R, Redefining the cerebellar cortex as an assembly of non-uniform Purkinje cell microcircuits. Nat. Rev. | Neurosci. 16, 79 (2015).

18. Demilly A, Reeber SL, Gebre SA, Sillitoe RV, Neurofilament Heavy Chain Expression Reveals a Unique Parasagittal Stripe Topography in the Mouse Cerebellum. The Cerebellum. 10, 409-421 (2011). [PubMed: 20127431]

19. Brochu G, Maler L, Hawkes R, Zebrin II: A polypeptide antigen expressed selectively by purkinje cells reveals compartments in rat and fish cerebellum. J. Comp. Neurol. 291, 538-552 (1990). [PubMed: 2329190]

20. Maekawa K, Simpson JI, "Climbing Fiber Responses Evoked in Vestibulocerebellum of Rabbit From Visual System" (1972), (available at www.physiology.org/journal/jn).

21. Wylie DRW, Brown MR, Winship IR, Crowder NA, Todd KG, Zonal organization of the vestibulocerebellum in pigeons (Columba livia): III. Projections of the translation zones of the ventral uvula and nodulus. J. Comp. Neurol. 465, 179-194 (2003). [PubMed: 12949780]

22. Chung C et al., Heat Shock Protein Beta-1 Modifies Anterior to Posterior Purkinje Cell Vulnerability in a Mouse Model of Niemann-Pick Type C Disease. PLoS Genet. 12, 1-22 (2016).

23. Stoodley CJ, Schmahmann JD, Evidence for topographic organization in the cerebellum of motor control versus cognitive and affective processing. Cortex. 46, 831-44 (2010). [PubMed: 20152963]

24. Carter RA et al., A Single-Cell Transcriptional Atlas of the Developing Murine Cerebellum. Curr. Biol. 28, 2910-2920.e2 (2018). [PubMed: 30220501]

25. Storer PD, Jones KJ, Ribosomal RNA transcriptional activation and processing in hamster rubrospinal motoneurons: Effects of axotomy and testosterone treatment. J. Comp. Neurol. 458, 326-333 (2003). [PubMed: 12619068]

\section{Supplemental References:}

26. Adams KL, Gallo V, The diversity and disparity of the glial scar. Nat. Neurosci. (2017), doi: 10.1038/s41593-017-0033-9.

27. Kenney AM, Kocsis JD, Peripheral axotomy induces long-term c-Jun amino-terminal kinase-1 activation and activator protein-1 binding activity by c-Jun and junD in adult rat dorsal root ganglia In vivo. J. Neurosci. 18, 1318-28 (1998). [PubMed: 9454841]

28. Robinson GA, Immediate early gene expression in axotomized and regenerating retinal ganglion cells of the adult rat. Mol. Brain Res. 24, 43-54 (1994). [PubMed: 7968376]

29. Honkaniemi J, Sagar SM, Pyykönen I, Hicks KJ, Sharp FR, Focal brain injury induces multiple immediate early genes encoding zinc finger transcription factors. Mol. Brain Res. 28, 157-163 (1995). [PubMed: 7707870]

30. Lowe DG, in Proceedings of the Seventh IEEE International Conference on Computer Vision (IEEE, 1999; http://ieeexplore.ieee.org/document/790410/), pp. 1150-1157 vol.2.

31. Patro Rob, Duggal Geet, Love Michael I., Irizarry Rafael A., and Kingsford Carl. 2017 "Salmon Provides Fast and Bias-Aware Quantification of Transcript Expression.” Nature Methods 14 (4): 417-19. [PubMed: 28263959]

32. Thevenaz P, Ruttimann UE, Unser M, A pyramid approach to subpixel registration based on intensity. IEEE Trans. Image Process. 7, $27-41$ (1998). [PubMed: 18267377] 
33. Kong Qiongman, Stockinger Michael P., Chang Yueming, Tashiro Hirofumi, and Lin Chien-Liang Glenn. 2008 "The Presence of rRNA Sequences in Polyadenylated RNA and Its Potential Functions.” Biotechnology Journal 3 (8): 1041-46. [PubMed: 18683164]

34. Müller D et al., Dlk1 Promotes a Fast Motor Neuron Biophysical Signature Required for Peak Force Execution. Science. 343, 1264-1266 (2014). [PubMed: 24626931]

35. Xiao J et al., Systematic regional variations in Purkinje cell spiking patterns. PLoS One. 9, e105633 (2014).

36. Zhou H et al., Cerebellar modules operate at different frequencies. 3, 2536 (2014).

37. Womack MD, Khodakhah K, Dendritic Control of Spontaneous Bursting in Cerebellar Purkinje Cells. J. Neurosci. 24, 3511-3521 (2004). [PubMed: 15071098]

38. Kim CH et al., Lobule-specific membrane excitability of cerebellar Purkinje cells. J. Physiol. 590, 273-288 (2012). [PubMed: 22083600] 
A Bead deposition In situ indexing

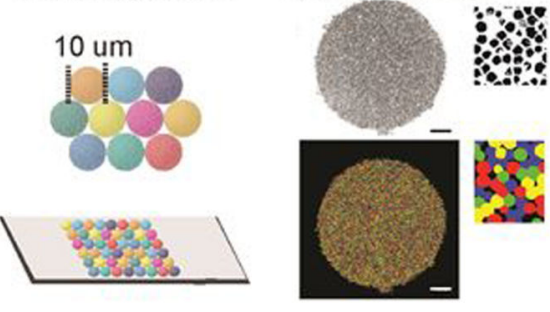

C

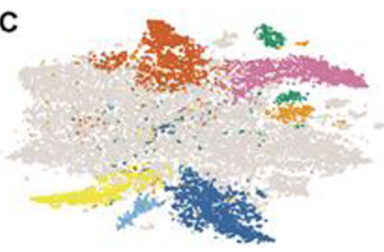

Fibroblast Ependymal Choroid Habenula Oligo

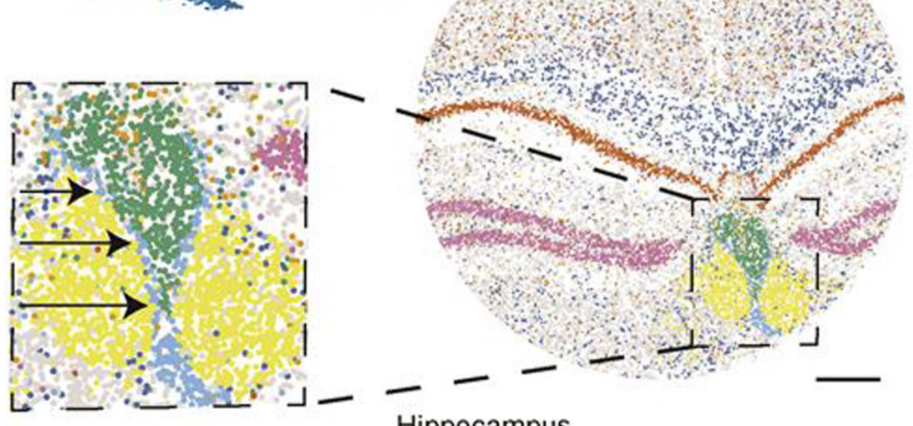

Hippocampus
B

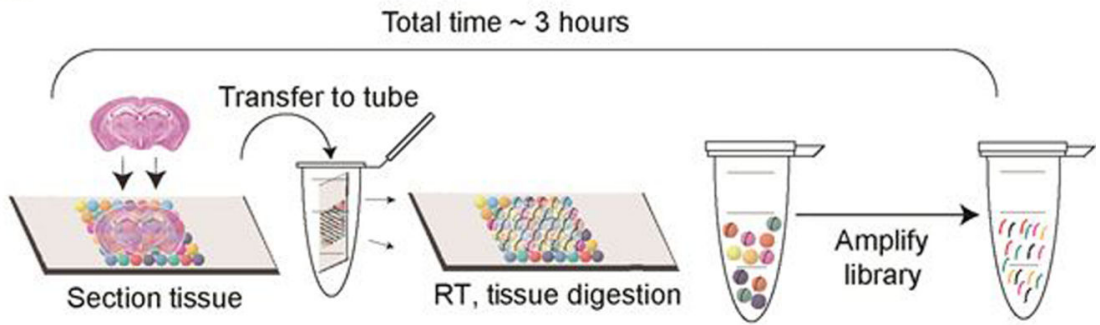

Figure 1: High-resolution RNA capture from tissue by Slide-seq.

(A) Left: Schematic of array generation. A monolayer of randomly deposited, DNA barcoded beads (termed a "puck") is spatially indexed by SOLiD sequencing. Top Right: A representative puck with sequenced barcodes shown in black. Bottom Right: A composite image of the same puck colored by the base calls for a single base of SOLiD sequencing. (B) Schematic of the sample preparation procedure developed for Slide-seq. (C) Top left: tSNE representation of Slide-seq beads from a coronal mouse hippocampus slice with colors indicating clusters. Right: the spatial position of each bead is shown, colored by the cluster assignments shown in the tSNE. Bottom left: Inset indicating the position of a single-cellthickness ependymal cell layer (black arrow). (D) As in (C), but for the indicated tissue type (see Fig. S2 for clustering and cluster identities). All scale bars $500 \mu \mathrm{m}$. 
A

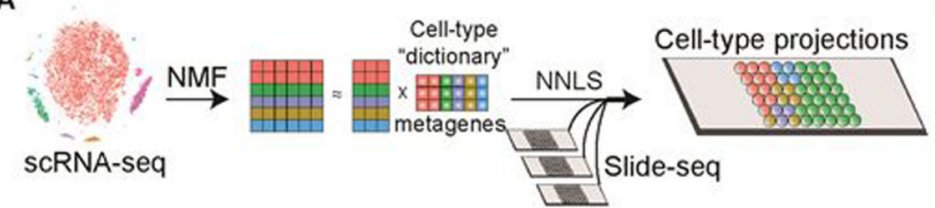

B Granule

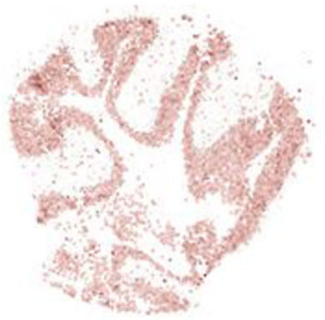

C $\quad 0.7$
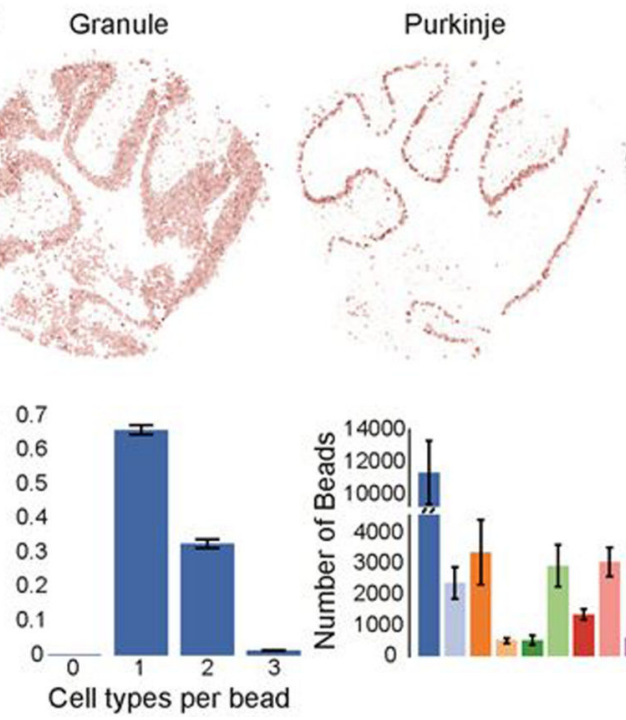

Unipolar Brush
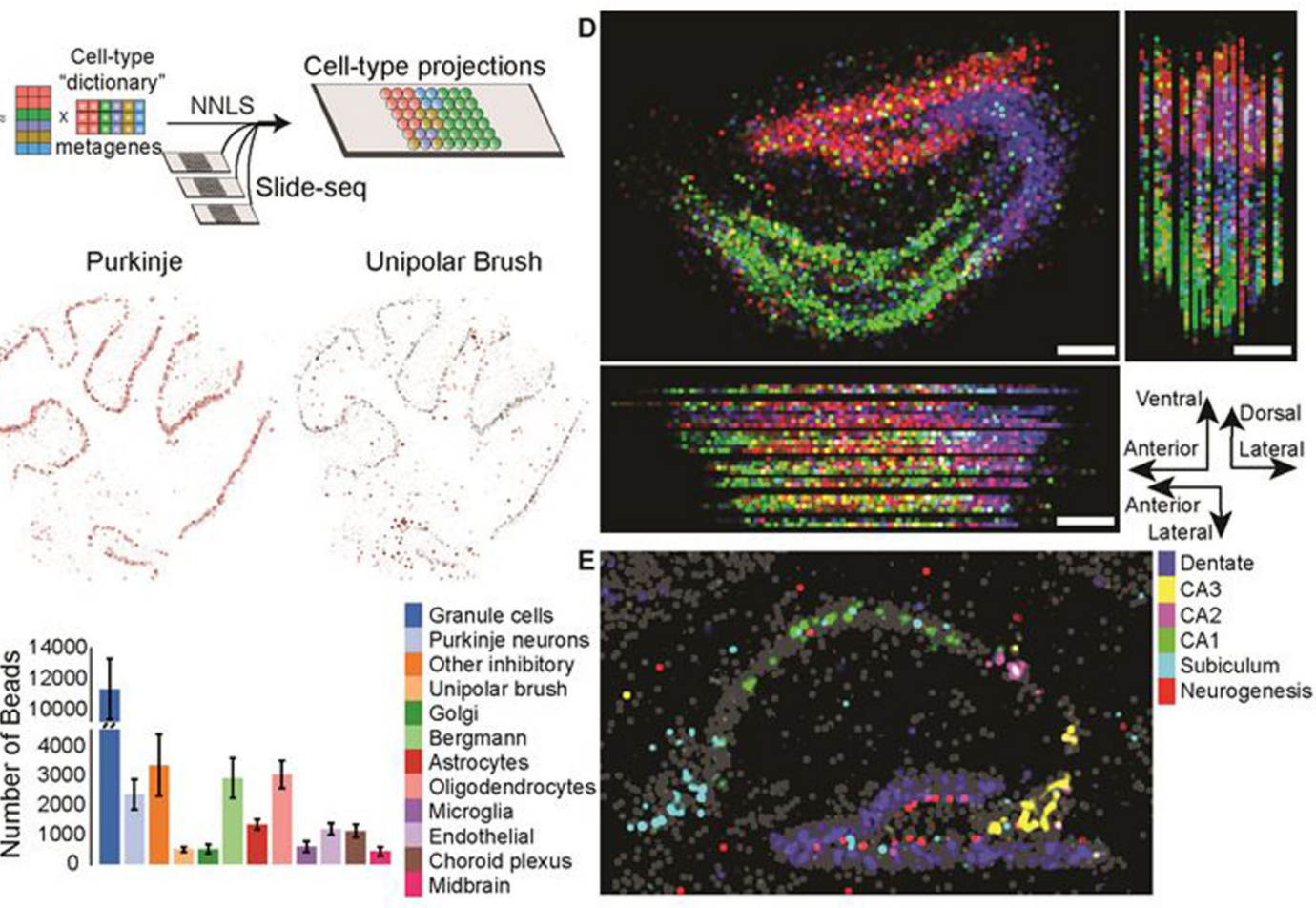
Dentate CA3

CA2

Subiculum Neurogenesis

Figure 2: Localization of cell types in cerebellum and hippocampus using Slide-seq.

(A) Schematic for assigning cell types from scRNA-seq datasets to Slide-seq beads using NMF and NNLS regression (NMFreg). (B) Loadings of individual cell types, defined by scRNA-seq cerebellum (9) on each bead of one $3 \mathrm{~mm}$-diameter coronal cerebellar puck (red, cell type location, gray, Purkinje loadings plotted as a counterstain). Other cell types are in Fig. S6. (C) Left: Number of cell types assigned per bead (Fig. S3). Right: The number of beads called as each scRNAseq-defined cell type for cerebellar pucks (mean \pm std. $\mathrm{N}=7$ pucks). (D) Projections of hippocampal volume with NMFreg cell type calls for CA1 (green), CA2/3 (blue) and dentate gyrus (Red). Top left: Sagittal projection. Top right: Coronal projection. Bottom left: Horizontal projection. Bottom right: axis orientations for each of the projections. (E) Composite image of metagenes for six different cell types. All scale bars $250 \mu \mathrm{m}$. All metagenes are listed in Table S2. 

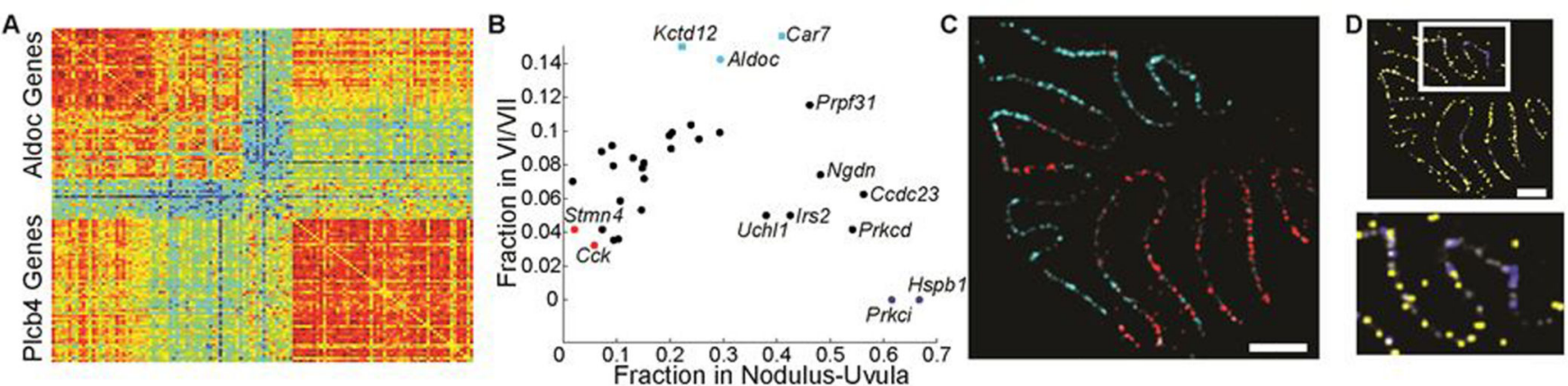

Figure 3: Identification of novel variation in cerebellar gene expression by Slide-seq.

(A) Heatmap illustrating the separation of Purkinje-expressed genes into two clusters by spatial gene correlation. The $i$, th entry is the number of genes found to overlap with both gene $i$ and $j$ in the Purkinje cluster (6). (B) For genes with significant expression $(p<0.001$, Fisher exact test) in the nodulus-uvula region (6), the fraction of reads localized to the nodulus/uvula and to the VI/VII boundary is shown. Pcp4, a ubiquitous marker for Purkinje cells, is in gray. (C) An Aldoc metagene in cyan. A Cck metagene in red. (D) A H2-D1 metagene in yellow. A Hspb1 metagene in blue. All scale bars show $250 \mu \mathrm{m}$. All metagenes are listed in Table S2. 
A

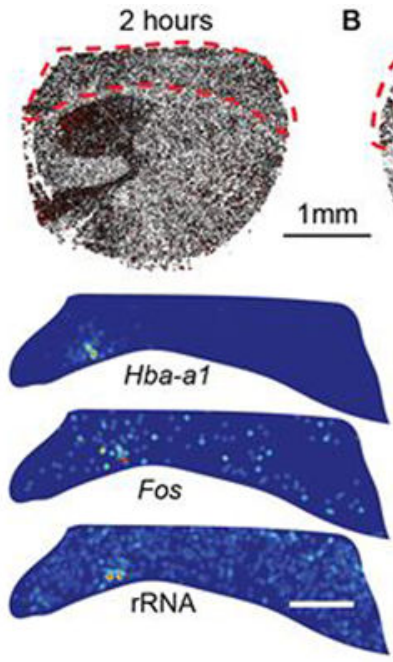

D

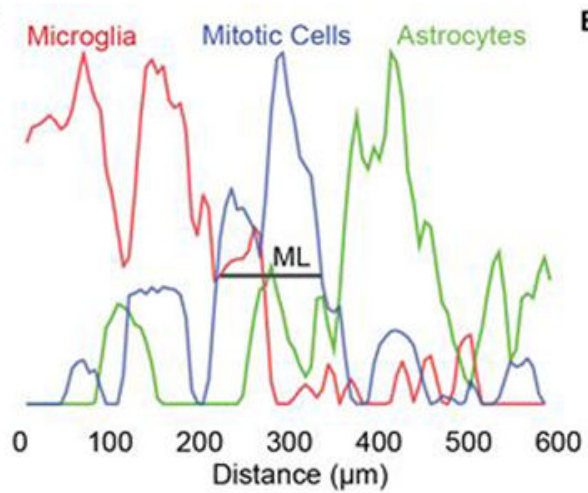

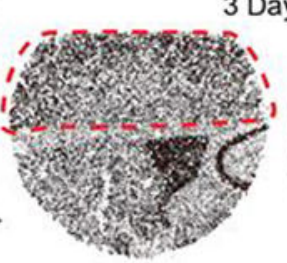
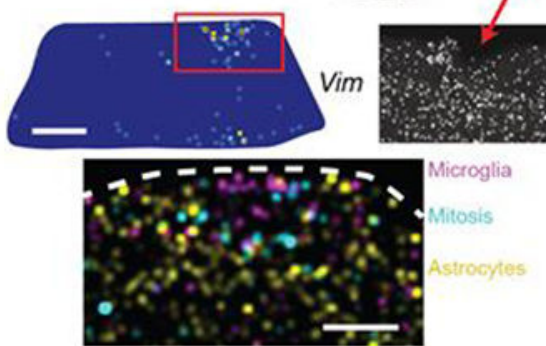

C

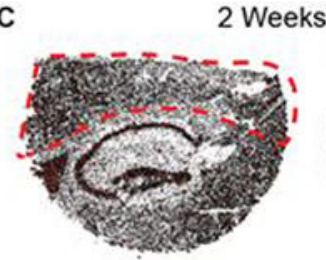

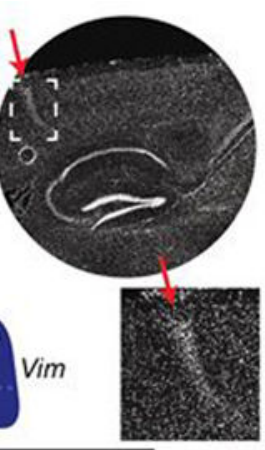
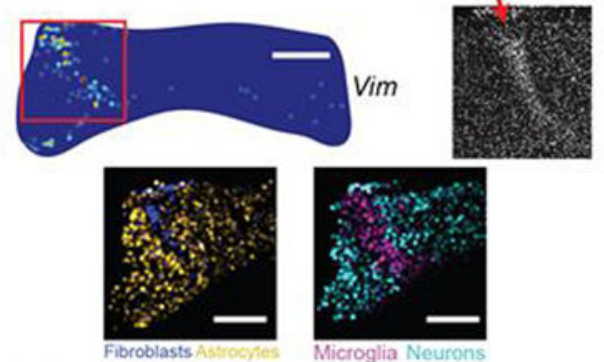

E

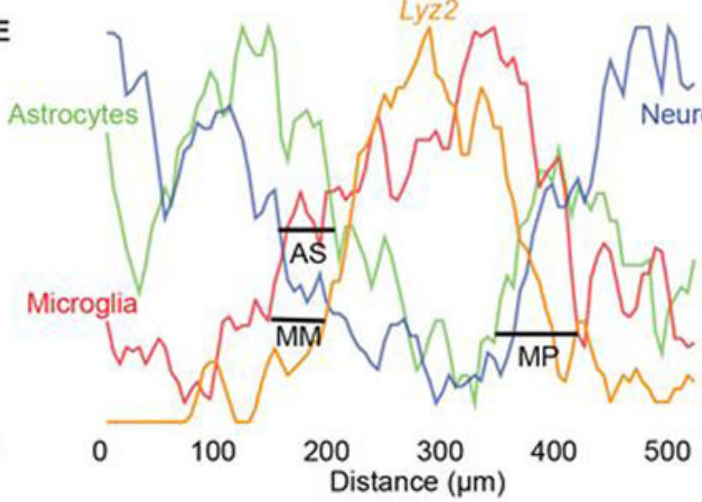

F 100

eurons

80

空 60

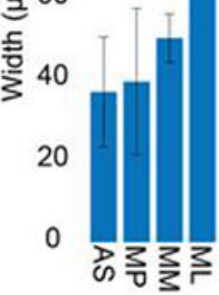

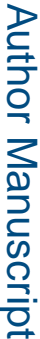

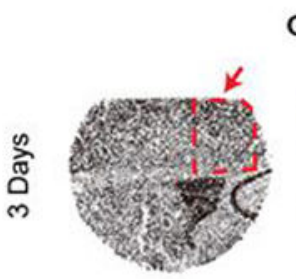

G Mitosis

$$
\text { H Antigen }
$$

'Gliogenesis

$J$ Oligo

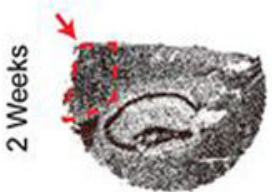

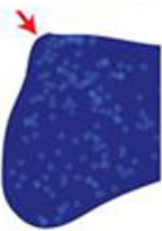

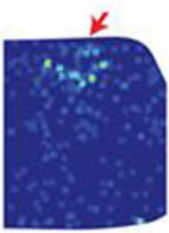

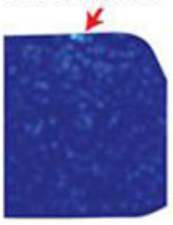
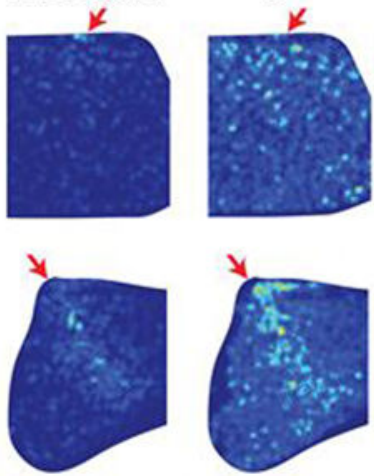

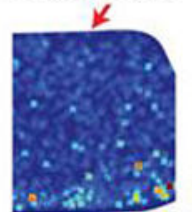

K

2 Weeks

IEG
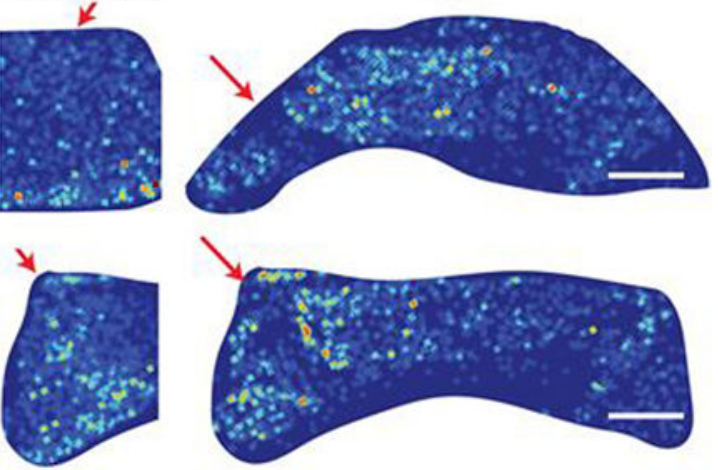

Figure 4: Slide-seq identifies local transcriptional responses to injury:

(A) Top: All mapped beads for a coronal hippocampal slice from a mouse sacrificed 2 hours after injury, with circle radius proportional to transcripts. Bottom: genes marking the injury. (B) As in (A), for a mouse sacrificed 3 days after injury. Top and middle right: DAPI image of an adjacent slice. Panels with black backgrounds show NMFreg cell types as density plots. Scale bar: $250 \mu \mathrm{m}$ (6). (C) As in (B), for a mouse sacrificed 2 weeks following injury. Bottom scale bar: $500 \mu \mathrm{m}$. (D) Spatial density profiles for the puck in (B) (6). (E) Spatial density profiles for the puck in (C). $L y z 2$ is plotted as a marker of macrophages. The vertical 
axis in (D) and (E) represents cell-type density in arbitrary units (6). (F) The thickness of the features in (D) and (E) (mean \pm std., $\mathrm{N}=6$ for scar, $\mathrm{N}=6$ for penetration, $\mathrm{N}=3$ for mitosis layer). (G-J) Gene ontology-derived metagenes for the puck in (B) (top) or (C) (bottom). Warmer colors correspond to greater metagene counts. (K) The IEG metagene (Table S2) for two 2-week pucks. Circular images in (A-C) refer to the scale bar in (A). All scale bars for images with blue backgrounds $500 \mu \mathrm{m}$. Red arrows indicate the injury. 